\title{
Spare Parts Requirements for Space Missions with Reconfigurability and Commonality
}

\author{
Afreen Siddiqi* and Olivier L. de Weck ${ }^{\dagger}$ \\ Massachusetts Institute of Technology, Cambridge, Massachusetts 02139
}

DOI: $10.2514 / 1.21847$

\begin{abstract}
The logistics resources (in the form of spare parts) for future exploration missions to the moon and Mars can have significant mass and volume requirements. In this study it is shown that reconfigurable or common parts that allow use of the same component in different mission elements, can be a means of reducing the required number of spares. A model for determining the system availability as a function of spares level is developed for a mission that utilizes elements with reconfigurable or common parts. An explicit consideration of the operating scenario is made that allows the use of components from elements that are not operating at a given time. A Mars surface exploration mission is used to illustrate the application of this model. It is shown, for the specific example studied in the analysis, that the same level of system availability can be achieved with 33-50\% fewer spares if the parts are reconfigurable or common across different mission elements.
\end{abstract}

\section{Nomenclature}

\begin{tabular}{|c|c|c|}
\hline$A\left(t_{i}\right)$ & $=$ & availability at $t_{i}$ \\
\hline $\bar{B}\left(t_{i}\right)$ & $=$ & expected backorder level at $t_{i}$ \\
\hline $\bar{B}_{c}\left(s, t_{i}\right)$ & $=$ & expected backorder level at $t_{i}$ for spares level $s$ \\
\hline E & $=$ & number of total elements in the mission \\
\hline$l$ & $=$ & failure rate (failures per day) \\
\hline$N$ & $=$ & $\begin{array}{l}\text { total number of units among all elements and } \\
\text { including spares repository }\end{array}$ \\
\hline$P(s)$ & $=$ & probability of having $s$ spares available \\
\hline$p(n)$ & $=$ & probability of $n$ failures \\
\hline$q_{e}$ & $=$ & no. of units of a component in element $e$ \\
\hline$s$ & $=$ & total number of spares \\
\hline$s_{E}$ & $=$ & $\begin{array}{l}\text { maximum number of spares available from idle } \\
\text { elements }\end{array}$ \\
\hline$s_{I}$ & $=$ & initial number of spares in repository \\
\hline$t_{i}$ & $=$ & $\begin{array}{l}\text { time instances at which operating elements change } \\
\text { their state }\end{array}$ \\
\hline$\Gamma_{e}\left(t_{k}\right)$ & $=$ & $\begin{array}{l}\text { binary variable with value } 1 \text { if element } e \text { is } \\
\text { operating in interval } t_{k}\end{array}$ \\
\hline $\begin{array}{l}\gamma\left(t_{i}\right) \\
\lambda\end{array}$ & $\begin{array}{l}= \\
=\end{array}$ & $\begin{array}{l}\text { percentage of elements operating at } t_{i} \\
\text { mean number of failures }\end{array}$ \\
\hline
\end{tabular}

\section{Introduction}

$\mathbf{F}$ UTURE human missions to the moon and Mars can be expected to use several elements. Typical elements comprising a surface mission may include ascent/descent vehicles, rovers for surface mobility, and habitats for long duration surface stays (see Fig. 1).

In such large missions, the required logistics resources can become significant. Common or reconfigurable spare parts, which can be configured to function in different systems as required, can potentially be of great benefit. The mass and volume of logistics resources (spare parts) needed for an exploration mission can be

Received 16 December 2005; revision received 9 March 2006; accepted for publication 9 March 2006. Copyright ( 2006 by Olivier de Weck and Afreen Siddiqi. Published by the American Institute of Aeronautics and Astronautics, Inc., with permission. Copies of this paper may be made for personal or internal use, on condition that the copier pay the $\$ 10.00$ per-copy fee to the Copyright Clearance Center, Inc., 222 Rosewood Drive, Danvers, MA 01923; include the code $\$ 10.00$ in correspondence with the CCC.

${ }^{*}$ Graduate Student, Department of Aeronautics \& Astronautics, Room 33409, AIAA Student Member.

Assistant Professor of Aeronautics \& Astronautics and Engineering Systems, Department of Aeronautics \& Astronautics, Engineering Systems Division, Room 33-410,77 Massachusetts Avenue, deweck@ mit.edu, AIAA Senior Member. greatly reduced through the use of such components. Furthermore, other maintenance and operation procedures can become easier due to commonality of components in different elements.

This paper develops a model for estimating the requirements of reconfigurable spare parts needed for systems that may not be operating concurrently. It then explores the amount of benefit that can be achieved in logistical costs through this reconfigurability. A key consideration made in the model is that of the operational scenario of the various elements. It is assumed that all elements involved in the mission do not necessarily operate simultaneously, for example, the ascent/descent vehicle and all the rovers do not have to operate at the same instance of time in the mission. A greater reuse of components therefore becomes possible. It should be noted, however, that the elements between which the parts are exchanged have to be in close proximity. In such a case, a functional part from a nonoperating (or idle) element can thus be borrowed or scavenged for installation on an operating element that has experienced a failure.

It is also assumed that there is a set of spare parts that are brought in with the mission and are utilized first as failures occur. The parts from idle elements are only reused on other operating elements if no new spares are available. The interchange of parts does not take place routinely, rather it is done as a last resort.

It should be noted that the case of commonality, that is, common parts among different elements is implicitly included in this work. The difference between the reconfigurable case and the common case is simply that in the former the parts will need to undergo a reconfiguration in some fashion before being installed on another element, while in the latter (i.e., in the commonality case) the parts can simply be used as is. So the same model applies to both cases, and the difference can manifest itself in the form of altered failure rates of the components or repair and service times. In general, it can be expected that in the reconfigurable case the failure rates and service times will be comparatively higher than the commonality case, however, this may not always be true.

Literature review: Several models have been produced for analyzing spare parts inventory requirements. Caglar and SimchiLevi [1] have shown how a two-echelon spares parts inventory system can be modeled and optimally solved for a given service time constraint. Shishko [2] has modeled costs of spares and repairs in estimating operations costs for the International Space Station. Bachman and Kline [3] have developed a model for estimating spare parts requirements for space exploration missions.

The model presented in [3] is used in this work as a base case (which is referred to as the "dedicated case" in this study) in which components are assumed to be used for only particular elements. An enhanced model is then formulated in which the parts can be assumed 


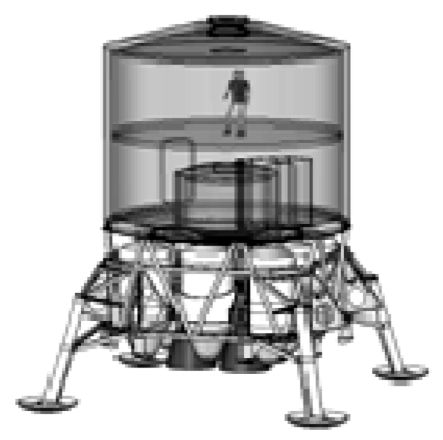

a)

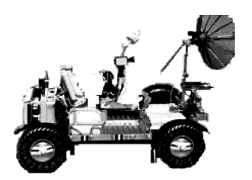

a) Surface habitat (HAB)

b) Ascent/descent vehichle (ADV) c) All terrain vehicle (ATV) c)

Fig. 1 Typical space exploration mission elements.

to be reconfigurable (or common) and thus an interchange of parts between mission elements subject to their operational state is permitted. The overall system availability is then formulated and an explicit provision of using components from idle elements is included. A sensitivity analysis is also performed for some limited cases to assess the impact of various parameters (such as component reliability and the operational scenario) on system availability.

\section{Model for Estimating Requirements of Reconfigurable Spare Parts}

A total of $E$, colocated elements are considered to be part of the mission. Although each element is composed of several subsystems and parts, for simplicity only one type of part is considered first here (the extension for several parts is discussed at the end of the section). It is assumed that there is no resupply (which is conceivable for a mission to Mars for instance), so no additional parts can be made available during the course of the mission. Furthermore, no repair capability is assumed. Thus, once a part fails it cannot be employed for use at a later time.

Figure 2 illustrates the usage model assumed for dedicated and reconfigurable parts. It shows that if elements are using dedicated components, then only particular spares designed for a particular element can be used from a spares repository. On the other hand, operating elements in need of reconfigurable (or common) spares can obtain the parts from either a spares repository or an idle element (as indicated with the dashed oval).

In the following sections a model for estimating spare parts requirements for elements using dedicated components is first discussed, and then a model in which reconfigurable/common spare parts are used is developed in an analogous fashion.

\section{A. Dedicated Spare Parts}

In this case the component used in each element will be for the dedicated use of only that element. For simplicity, consider that there is only one type of component installed on element $j$ (Fig. 2a). The quantity per application (QPA), which is the number of units of the component used, will be denoted as $q_{j}$. It is assumed that there is a spare parts repository which is a collection of spares for the various mission elements. The spares level for each element's component in that repository is $s_{j}$. The sum of all $s_{j}$ is $s_{I}$ which is the total number of spares initially in the repository at the start of the mission.

It is considered that the components fail according to a Poisson process described by

$$
p_{j}(n)=\frac{e^{-\lambda_{j}} \lambda_{j}^{n}}{n !}
$$

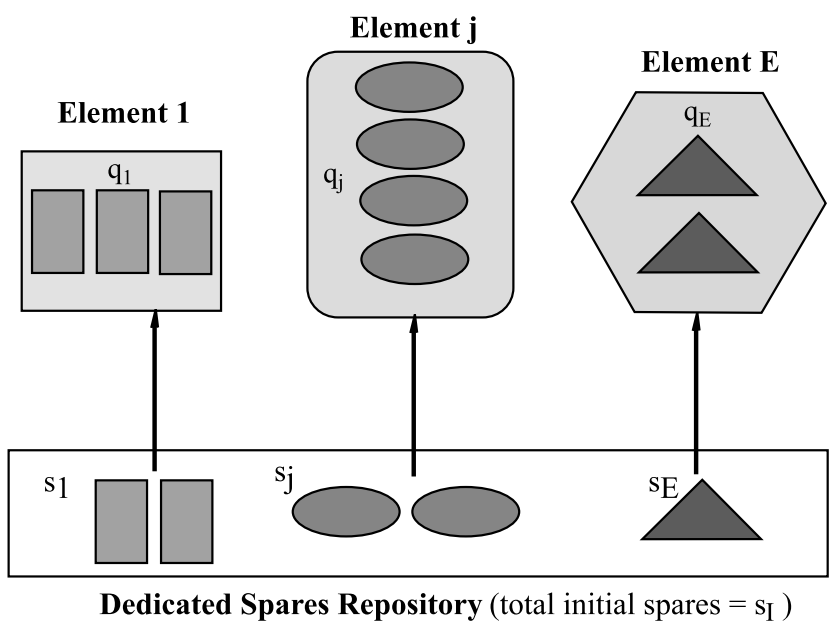

a) Dedicated spares for particular elements

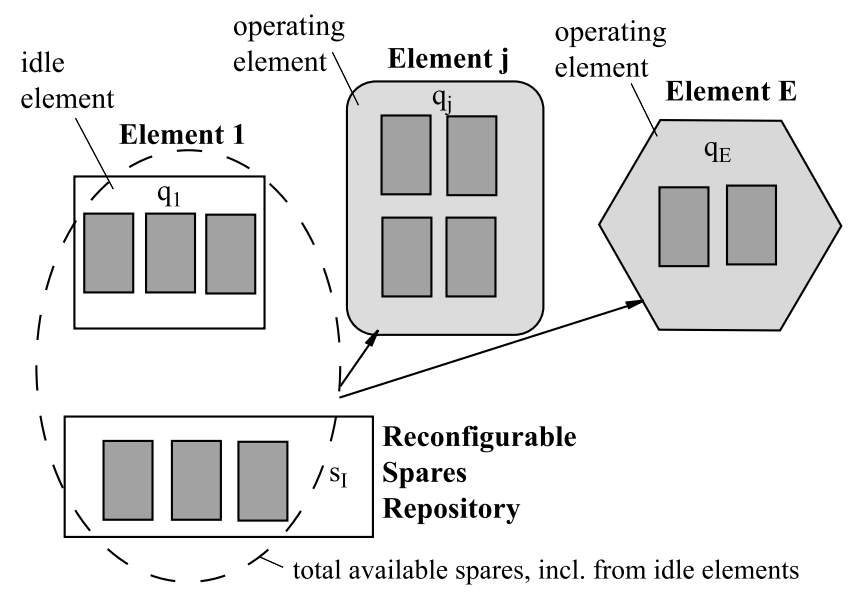

b) Reconfigurable spares for any element

Fig. 2 Dedicated and reconfigurable spares usability by elements.

$$
\lambda_{j}=\int_{t_{o}}^{t_{f}} l_{j} \mathrm{~d} t
$$

where $p_{j}(n)$ is the probability of having exactly $n$ failures of the component in element $j$ in interval $\left[t_{o} t_{f}\right]$ for a process that has a failure rate of $l_{j}$, which is the inverse of its mean time to failure (MTTF). The mean of such a Poisson process can be shown to be equal to $\lambda_{j}$. If there are $q_{j}$ units, and $l_{j}$ is independent of time, then the mean number of failures in interval $\left[t_{o} t_{f}\right]$ is simply

$$
\lambda_{j}=q_{j} l_{j}\left(t_{f}-t_{o}\right)
$$

and is sometimes referred to as "outstanding orders" [1], or as the "number in the pipeline" [3]. Note that the interval $\left[t_{o} t_{f}\right]$ is the duration the component has operated for. The expected backorder level, $\bar{B}_{j}$, is the expected value of the difference between the actual number of spares that are available and the required number of spares (as driven by the number of failed components). It is therefore computed as [3]

$$
\bar{B}_{j}=\sum_{n>s_{j}}^{\infty}\left(n-s_{j}\right) p_{j}(n)
$$

In reality the upper bound on the summation is the total failures that can occur of the component.

In this case redundancy in an element is ignored, and it is assumed that an element is operational if it does not have any outstanding requests for replacing any failed component, that is, its $\bar{B}$ is zero. If it 
is assumed that the backorders of the part are uniformly distributed across the elements, then the probability that there is an outstanding request for a component in a particular element is $\bar{B}_{j} / q_{j}$. The failures of the components in the different elements are considered to be independent, and thus the availability (of the element that uses this component) is

$$
A_{j}=\left(1-\frac{\bar{B}_{j}}{q_{j}}\right)^{q_{j}}
$$

It should be noted that $A$ is the availability that can be expected at the end of time $t_{f}$ since it was used for evaluating $\lambda$ given in Eq. (3). The system availability can be defined as the probability of all of the elements that should be operating to be actually operational. The overall availability is then simply the product of availability of all the elements (assuming that all the elements need to be fully operational for the entire mission)

$$
A_{\mathrm{sys}}=\prod_{j=1}^{E} A_{j}
$$

If more than one component needs to be considered in the analysis, this model is easily extended by using Eq. (5) for each component and then taking the product to get element availability (assuming that all of those components have to be operational for the element to be considered available). However, this can get more complex if redundancy is also modeled.

\section{B. Reconfigurable Spare Parts}

The above approach is the traditional analysis in which it was assumed that elements use dedicated components and hence have corresponding dedicated spares for maintenance. A spares inventory stock level for a repository can be estimated by analyzing failure rates and operation duration and other desired constraints (such as mean delivery times, etc.) for a mission.

Now consider the case where it is assumed that reconfigurable components are used, and a particular component can be either reconfigured (or utilized as is) for use on a variety of different elements in the mission (Fig. 2b). A specific instance of the component installed on one element that is not operating for a period of time during the mission can therefore be employed on a different element that may need it (which is also referred to as temporary scavenging or cannibalization) when all the spares from the inventory have been depleted. In such a scenario, an element $e$, during its idle time, that is, the time when it is not operating, can then be viewed as a potential spares repository that can supply a maximum of $q_{e}$ spares if required (where $q_{e}$ is the QPA of that component in element $e$ ). In such a situation (and assuming the elements are colocated) there is not just one warehouse of spares, but also other temporal repositories that can supply limited quantities of spares at certain points over the course of the mission. It needs to be emphasized that the existence of those repositories is a function of the mission time. Furthermore, the spares from these temporal repositories are not necessarily new, rather they may have operated for a period of time.

The system availability will be based on the number of potential spares that can be obtained from the initial spares repository as well as idle mission elements and will thus be affected by the operational scenario. The operating time profile of each element over the course of the mission therefore has to be first considered. Using this information, a set of time instances can then be defined:

$$
T=\left[t_{1}, \ldots, t_{i}, \ldots, t_{n}\right]
$$

where $t_{i}$ is a time instant in which there is a change in the operating mission elements. Note that the $t_{i}$ are not necessarily equally spaced in time, that is, $t_{i}-t_{i-1} \neq t_{i-1}-t_{i-2}$ for some $i$. Figure 3 illustrates this graphically where a few elements are shown with a specific operation profile. The status of each element is one when it is

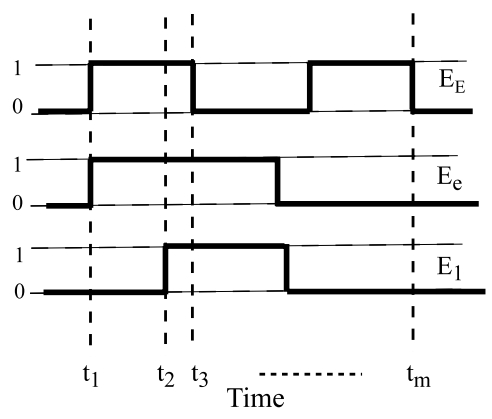

Fig. 3 Notional operational profile $\Gamma_{e}$ of various mission elements over time.

operating, and zero when it is idle. The instants where there is a change in the operating elements are marked as $t_{1}, t_{2}$, etc.

Assuming that each element comes initially equipped with its full set of functioning units of the component (meaning it has its $q_{e}$ units installed), the maximum number of spares that can be available from idle elements at time $t_{i}$ is then

$$
s_{E}\left(t_{i}\right)=\sum_{e=1}^{E} q_{e}\left[\neg \Gamma_{e}\left(t_{i}\right)\right]
$$

where $\Gamma_{e}\left(t_{i}\right)=1$ if element $e$ is operating in an interval starting at $t_{i}$ and is zero if idle. The symbol $\neg$ is for the logical NOT operation.

Note that the element is considered to be operating in this context if it carries out functions that require the component to be installed and is idle if it is in a state that does not require its presence. Thus, in reality each element can potentially have different $\Gamma$ for each component at any instant of time depending on its particular operational state (because all components may not be required for each state). Therefore, when several components are being considered $\Gamma$ should be determined on the component level for a better assessment of the impact of reconfigurablity (or commonality). Here, since only one type of part is being analyzed, the $\Gamma$ is taken at the element level.

It is assumed for simplicity that the failure rate is the same for all the reconfigurable components in the elements and is denoted as $l$. In more detailed analyses the failure rate is often adjusted for each particular element with various factors (so called $K$ factors [3]) that account for environmental and other relevant aspects. However, these are also ignored for simplicity here. For each element, $e$, the mean number of failures is then

$$
\lambda_{e}\left(t_{i}\right)=q_{e} l \sum_{k=1}^{i}\left[t_{k}-t_{k-1}\right] \Gamma_{e}\left(t_{k}\right)
$$

This is the mean number of failures the element has experienced over interval $\left[t_{o} t_{i}\right]$ based on its particular operational time (which gets accounted for through the use of $\Gamma$ ).

The above equation is applicable when the operational history of each component (or part) is equal to the operational history of the element to which it belongs. However, in the case when failed parts can be replaced by new spares in an inventory, then the computation of the mean number of failures of a part type using $q_{e}$ in the calculation will be conservative. This is because if we suppose that there is a fresh spare among the $q_{e}$ parts on an element, then $q_{e}-1$ parts have the same operation time as the element, while the new spare has operated for a shorter time. For an analysis in which only dedicated spares are considered along with the possibility of replacement with new spares, this is a reasonable way to compute the mean number of failures for a given duration of time.

In the situation when some parts are allowed to be cannibalized from other elements (after the new spares have been depleted), then a particular part on an element may have operated for a longer period of time than the element it may temporarily be installed in. Figure 4 shows the three types of parts (based on how their operational history compares with that of the element on which they are installed). 


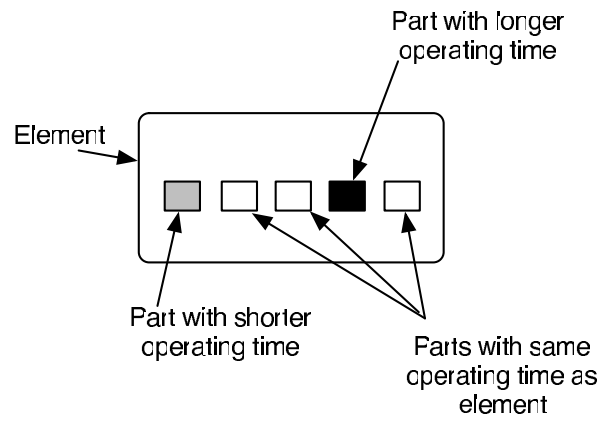

Fig. 4 Types of parts that can exist in an element.

In this case, Eq. (9) is appropriate if the cannibalization would occur very rarely. In that situation, the possibility of having a part on an element that has operated longer than the element it is installed on will be very low, and Eq. (9) can still be applicable with some degree of caution. However, if the cannibalization can happen frequently (perhaps due to a combination of high failure rates and fewer new spares, etc.), then the problem can be analyzed in two ways. It can either be simulated (in which the operational and element installation history of each part will be known explicitly), or a more conservative estimate of the mean failures is made by noting that the operation time of any part at a time instance cannot exceed the total mission time elapsed up to that time. In that case

$$
\lambda_{e}\left(t_{i}\right)=q_{e} l \sum_{k=1}^{i}\left[t_{k}-t_{k-1}\right]
$$

Note that this bound can give significantly conservative results if the operation duration of an element is much less than the total mission time. However, this simplification allows an analytical assessment (instead of simulation) when the chances of cannibalization are great enough that the subsequent effect on the part failure rate cannot be ignored.

The mean number of failed components of a particular type from all the elements in interval $\left[t_{o} t_{i}\right]$ is

$$
\lambda\left(t_{i}\right)=\sum_{e=1}^{E} \lambda_{e}\left(t_{i}\right)
$$

If the total number of failures (which is a random variable) up to a given time instance is $n_{F}$, then the number of total spares (which is also a random variable) is

$$
s\left(t_{i}\right)=s_{I}+s_{E}\left(t_{i}\right)-n_{F}
$$

The term $s_{I}$ denotes the initial quantity of spares in an actual repository, whereas $s_{E}\left(t_{i}\right)$ as described earlier is the additional spares that can be provided by the various idle elements (which act as pseudorepositories). If $n_{F}>s_{I}+s_{E}\left(t_{i}\right)$, then $s\left(t_{i}\right)$ is zero.

The number of failures $n_{F}$ can assume integer values ranging from 0 to $N$, where $N$ is the total units of the component in the mission

$$
\begin{aligned}
& N=s_{I}+Q \\
& Q=\sum_{e=1}^{E} q_{e}
\end{aligned}
$$

The random variable $s\left(t_{i}\right)$ can also take integer values that are according to Eq. (12). For zero failures it can have a maximum value of $s_{I}+s_{E}\left(t_{i}\right)$, or it can have a minimum value of zero if no spares are available.

In the dedicated case each element has its own set of spares, and therefore the spares level $s_{j}$ for each element $j$ is used to determine its particular backorder level. In the augmented model with reconfigurable or common parts, because all the elements are supplied with replacement parts from a total of $s\left(t_{i}\right)$ spares, the backorder levels are computed at the mission level (across all elements). The expected backorder level at $t_{i}$, for the condition when the total spares level is $s$, is then

$$
\bar{B}_{c}\left(s, t_{i}\right)=\sum_{n_{F}=s+1}^{N}\left[n_{F}-s\right] p\left(n_{F}\right)
$$

The expected backorder level after accounting for all the possible values of $s$ is then

$$
\bar{B}\left(t_{i}\right)=\sum_{s=0}^{S} \bar{B}_{c}\left(s, t_{i}\right) P(s)
$$

$$
\bar{B}\left(t_{i}\right)=\sum_{s=0}^{S}\left(P(s) \sum_{n_{F}=s+1}^{N}\left(n_{F}-s\right) p\left(n_{F}\right)\right)
$$

where $S$ is the maximum value of the total spares level at time $t$ (corresponding to zero failures). The probability $P(s)$ is equal to the probability of $n_{F}$ failures occurring which result in a spares level $s$ [as given by Eq. (12)]. For instance, if the possible values of $s\left(t_{i}\right)$ in an interval are 0,1 , and 2 , then the conditional backorder levels $\bar{B}_{c}(0)$, $\bar{B}_{c}(1)$, and $\bar{B}_{c}(2)$ which correspond to each possible value of $s$ are computed. The values are then multiplied with $P(0), P(1)$, and $P(2)$ and summed to get the overall backorder level $\bar{B}\left(t_{i}\right)$.

The probability of a total of $n_{F}$ failures taking place up to a time $t_{i}$ in the mission across all the elements is computed by considering all the possible ways in which that number of failures can occur, for instance, if there are two mission elements and $n_{F}$ is 2 . There are three possible ways in which a total of two failures can occur. In the first case there can be two failures in the first element and none in the second, or in a second case there can be no failures in the first element and two in the second, or in the third case there can be one failure each in the two elements. In all these scenarios the total number of failures is the same; however, each individual element has a different number of failures. The number of failures that an element $e$ experiences in a particular scenario $w$ will be denoted as $n_{e}^{w}$. The probability of any individual scenario $w$ occurring is the product of the probabilities of the specific failures occurring in each element in that particular scenario (assuming the failures are independent).

$$
P_{w}=\prod_{e=1}^{E} p\left(n_{e}^{w}\right)
$$

For instance, in the example described above, the probability of the first scenario taking place is the product of the probability of having two failures in the first element and the probability of having no failures in the second element. The probability of having $n_{e}^{w}$ failures in element $e$ is the Poisson probability

$$
p\left(n_{e}^{w}\right)=\frac{e^{-\lambda_{e}} \lambda^{n_{e}^{w}}}{n_{e}^{w} !}
$$

The argument $t_{i}$ has been omitted from $\lambda_{e}$ for simplicity here. The probability of having $n_{F}$ failures occur across all the mission elements is then the sum of probabilities of all the various ways in which it can happen. Therefore

$$
p\left(n_{F}\right)=\sum_{w=1}^{W} P_{w}
$$

where $W$ is the number of all the combinations in which a total of $n_{F}$ failures can occur from among $E$ elements with a total of $Q$ components. It should be noted that $P(s)$ can be computed in a similar fashion as $p\left(n_{F}\right)$.

Using Eq. (17), the overall availability at $t_{i}$ is then simply

$$
A_{\mathrm{sys}}\left(t_{i}\right)=\left(1-\frac{\bar{B}\left(t_{i}\right)}{Q}\right)^{Q}
$$

It can be observed that now the end-of-mission availability is not 
directly relevant (which is usually the case in traditional studies). $A$ is no longer a monotonically decreasing function of time due to the explicit consideration of making use of components from other elements. The end-of-mission availability may be higher due to more spares being potentially available if fewer elements are operating, while the availability at some intermediate mission time maybe lower when more elements are operating at that time. The availability for each interval can be computed using the above equation. Because the worst case scenario is often of interest, the mission level availability for this reconfigurable spare parts case can then be taken as the minimum system availability that occurs at any time during the mission duration.

$$
A_{\mathrm{sys}}=\min \left[A_{\mathrm{sys}}\left(t_{i}\right)\right] \quad t_{i} \in T
$$

\section{Monte Carlo Simulation}

The validity of this model for reconfigurable or common spares can be tested through a discrete-event simulator. Furthermore, as discussed previously, a simulator can serve to assess the conservativeness of the assumptions regarding part operation times for Eq. (10).

In the simulation, the failure of parts installed on a set of given mission elements (Fig. 1) is simulated as a Poisson process (with the details as discussed in the previous section). Each part is allowed to be in one of three different states. It can either be operating (if it is on an operating element), it can be idle (if it is either installed on an idle element, or is in the spares repository), or it can be in a failed state. Once the part has failed, it cannot be repaired.

If a part fails, it is replaced immediately with a spare part from the repository if one is available. These spares are new and therefore have not operated for any duration of time until they are installed on an element. Their probability of failure at any day will therefore be different (and lower) than the failure probability of other parts that have been working in the element for longer periods of time. Note, that as failures occur during the mission, the fresh spares from the repository are first used. If there are more failures and the spares repository is empty, only then parts from idle elements are taken away to be installed on operating elements that need them. The cannibalization of idle elements is thus done as a last resort when all the new spares have been used up. In the simulation the cannibalization is done randomly from the first idle part that is found. In a more realistic case, priorities can be assigned to elements so that relatively less critical elements can be more readily cannibalized than the more critical ones (because cannibalization can lead to new problems and potential failures of other parts in the element).

If at any time instance, there are more spares required than are available, then the spares are allocated to the elements in an optimal way such that the mission level availability is maximized. Another algorithm for assigning the spares can be based on the priority level of the mission elements. The elements with higher priority (e.g., those that are critical for crew survivability, etc.) can be assigned spares before low priority elements are given the leftover parts.

For each simulation, a uniformly distributed random number is generated which is then used to determine if a part fails or not at each time step (e.g., each day of a mission). A part is allowed to fail if its probability of failure is greater than the random number, otherwise it is allowed to continue operation and its operational time is incremented by one time step.

If there is a failure, the part is replaced with either new or used spares according to the procedure described above. If there are no parts that can be installed in place of the failed one, the element on which the part failed is considered to be unavailable or down.

Even if a failure does not occur in a particular time step, but an element is down (when it should be operational), the availability of spares is checked and appropriate assignments are made if possible. Because the spare parts are a function of time due to the operational scenario of the elements, an element may be down due to lack of spare parts for some duration of the mission, but it may be able to function again later on if some other element becomes idle and its parts become available for temporary cannibalization.

Each simulation run of a specific mission (over a certain mission time line) plays out one particular outcome that can unfold. Hundreds of such simulations therefore need to be carried out so that a statistical analysis can be done to assess how the availability of the elements compares with the analytical predictions. Section V will discuss the results of a case study, in which the model predictions and simulated results will be compared for a sample exploration mission.

\section{Sensitivity Analysis}

It can be seen that the system availability [Eq. (22)] is a function of failure rate $l$, QPA, operational scenario $\Gamma$, and spares level, $s_{I}$. For a given value of $s_{I}$ (which are the number of spares brought initially at the start of the mission), it can be useful to assess how the availability varies with the other parameters.

\section{A. Availability and Quantity}

For finite values of $q$, Eq. (5) can be used for determining how $A$ changes with $q$. However, using the general relation (for a variable $x$ )

$$
\lim _{n \rightarrow \infty}\left(1+\frac{x}{n}\right)^{n}=e^{x}
$$

it can be seen that if $q$ becomes large (i.e., many units of the same component are used) then the availability is simply an exponential function of the backorder level.

$$
\begin{aligned}
\lim _{q \rightarrow \infty} A & =\lim _{q \rightarrow \infty}\left(1+\frac{-\bar{B}}{q}\right)^{q} \\
& =e^{-\bar{B}}
\end{aligned}
$$

Thus, availability can decrease extremely rapidly if the backorder level makes even a modest climb.

\section{B. Availability and Failure Rate}

\section{Dedicated Case}

For the case of dedicated components, the availability of an element is given by Eq. (5) which is reproduced below.

$$
A=\left(1-\frac{\bar{B}}{q}\right)^{q}
$$

The subscripts $i$ and $e$ have been dropped because we are considering only one element and one component in that element. The derivative of $A$ with respect to the failure rate $l$ can be expressed as

$$
\begin{aligned}
\frac{\mathrm{d} A}{\mathrm{~d} l} & =q\left(1-\frac{\bar{B}}{q}\right)^{q-1}\left(-\frac{1}{q} \frac{\mathrm{d} \bar{B}}{\mathrm{~d} l}\right) \\
& =q \frac{A}{(q-\bar{B}) / q}\left(-\frac{1}{q} \frac{\mathrm{d} \bar{B}}{\mathrm{~d} l}\right) \\
& =\frac{A q}{(\bar{B}-q)} \frac{\mathrm{d} \bar{B}}{\mathrm{~d} l} \\
& =\frac{A q}{(\bar{B}-q)}\left[\frac{\mathrm{d}}{\mathrm{d} l}\left(\sum_{n>s}(n-s) p(n)\right)\right]
\end{aligned}
$$

Since $l$ is the variable of interest, a constant $\alpha$ in this context can be defined

$$
\alpha=q \Delta t
$$

where $\Delta t$ is the total duration of time for which the element operated. The number in the pipeline is then 


$$
\lambda=\alpha l
$$

Equation (29) can then be written as

$$
\begin{aligned}
& \frac{\mathrm{d} A}{\mathrm{~d} l}=\frac{A q}{(\bar{B}-q)}\left[\sum_{n>s} \frac{(n-s)}{n !} \frac{\mathrm{d}}{\mathrm{d} l}\left\{e^{-\alpha l}(\alpha l)^{n}\right\}\right] \\
& \frac{\mathrm{d} A}{\mathrm{~d} l}=\frac{A q}{(\bar{B}-q)}\left[\sum_{n>s}(n-s) p(n)\left(\frac{n}{l}-\alpha\right)\right]
\end{aligned}
$$

As expected, the rate of change of $A$ with respect to $l$ is negative since $\bar{B}$ cannot be greater than $q$. Thus the availability decreases with increasing failure rate.

\section{Reconfigurable Case}

In the reconfigurable case, a similar procedure for determining the derivative from the availability equation can be followed. In this case it should be recalled that the number of failures and spares available was computed for all the elements combined at the mission level. Therefore to be able to compare the $\mathrm{d} A / \mathrm{d} l$ at the element level between the two cases, it is assumed for the reconfigurable case that the variable $s$ denotes the spares level that a particular element can use. Because the objective of this analysis is to simply compare the sensitivity expressions of availability with the failure rate, this assumption is reasonable in this context. If subscript $r$ is used for the reconfigurable case, then the following expression can be written for the availability of a particular element at time $t_{i}$ :

$$
\frac{\mathrm{d} A_{r}}{\mathrm{~d} l}=\frac{A_{r} q}{\bar{B}_{r}-q} \frac{\mathrm{d} \bar{B}_{r}}{\mathrm{~d} l}
$$

Using Eq. (17), the above expression can be written as

$$
\frac{\mathrm{d} A_{r}}{\mathrm{~d} l}=\frac{A_{r} q}{\bar{B}_{r}-q}\left[\sum_{s=0}^{S}\left(P(s) \sum_{n>s}(n-s) \frac{\mathrm{d}}{\mathrm{d} l} p(n)+\bar{B}_{c}(s) \frac{\mathrm{d}}{\mathrm{d} l} P(s)\right)\right]
$$

Using the development of Eq. (32) for the derivative of $p(n)$, the above equation becomes

$$
\begin{aligned}
& \frac{\mathrm{d} A_{r}}{\mathrm{~d} l}=\frac{A_{r} q}{\bar{B}_{r}-q} \\
& \quad \times\left\{\sum_{s=0}^{S}\left[P(s) \sum_{n>s}(n-s) p(n)\left(\frac{n}{l}-\alpha\right)+\bar{B}_{c}(s) \frac{\mathrm{d}}{\mathrm{d} l} P(s)\right]\right\}
\end{aligned}
$$

From the above equation and Eq. (33) it is sufficient to see that the rate of change of $A$ with respect to $l$ is different for the dedicated and reconfigurable cases. This difference in the sensitivities of $A$ to $l$ [as evident from Eqs. (33) and (36)] can become important in studying the tradeoffs between reconfigurable components and dedicated components versus different reliabilities. Section $\mathrm{V}$ provides an example of such a case.

\section{Availability and Operation Time}

The model developed for the reconfigurable case makes the backorder levels and thus availability dependent on the number of idle elements at a given instance of time. The operational scenario can greatly affect mission level availability. To assess the impact of operational times a quantity $\gamma$ can be defined such that it is the fraction of operating elements out of the total mission elements at a particular time. Thus

$$
\gamma_{i}=\frac{1}{E} \sum_{e=1}^{E} \Gamma_{e}\left(t_{i}\right)
$$

With this definition $\gamma$ varies between 0 (when no element is operating) and 1 (when all elements are operating simultaneously). If a simplifying assumption is made that all elements in the mission have the same QPA of the component, that is, $q_{e}=q \quad \forall e$, then the maximum number of spares that can be obtained from the elements $s_{E}$ at time $t_{i}$ [shown in Eq. (8)] is

$$
s_{E}\left(t_{i}\right)=q \sum_{e=1}^{E} \neg \Gamma_{e}\left(t_{i}\right)
$$

The summation term is simply the number of idle elements at $t_{i}$, so

$$
s_{E}\left(t_{i}\right)=q E\left(1-\gamma_{i}\right)
$$

From Eq. (12), it follows that the spares $s\left(t_{i}\right)$ available at instant $t_{i}$ is now also a function of $\gamma$ and is

$$
s\left(t_{i}, \gamma_{i}\right)=s_{I}+q E\left(1-\gamma_{i}\right)-n_{F}
$$

The first term is the initial inventory or fresh spares, the second term represents the pseudoinventory of spares from idle elements, and the third term in the sum is the total cumulative number of failed parts across all elements up to time $t_{i}$ in the mission. The derivative of the availability $A_{r}$ of an element with respect to $\gamma$ at time $t_{i}$ can now be expressed [in a similar fashion as Eq. (34) and with the same assumptions] as

$$
\frac{\mathrm{d} A_{r}}{\mathrm{~d} \gamma}=\frac{A_{r} q}{\bar{B}_{r}-q} \frac{\mathrm{d}}{\mathrm{d} \gamma} \bar{B}_{r}
$$

The above equation can be expanded further as

$$
\frac{\mathrm{d} A_{r}}{\mathrm{~d} \gamma}=\frac{A_{r} q}{\bar{B}_{r}-q}\left[\sum_{s=0}^{S} P(s) \frac{\mathrm{d}}{\mathrm{d} \gamma} \bar{B}_{c}(s)+\bar{B}_{c}(s) \frac{\mathrm{d}}{\mathrm{d} \gamma} P(s)\right]
$$

Now, in the dedicated case the availability is not dependent on which other elements are operating at a given instant of time. Therefore there is no dependence on $\gamma$ and

$$
\frac{\mathrm{d} A}{\mathrm{~d} \gamma}=0
$$

To show that the variation of the availability with $\gamma$ is different for the dedicated and reconfigurable/common case, it is sufficient to see that Eqs. (42) and (43) are not the same.

\section{Example: Mars Exploration Mission}

A case study of a planetary exploration mission can be developed for assessing the spare parts requirements for reconfigurable and also dedicated components. Furthermore, to test the validity of the model, Monte Carlo simulations are carried out using a discrete-event simulation of the mission.

A surface exploration mission of Mars is considered in which the mission elements are an ascent/descent vehicle (ADV), a surface habitat (HAB), an unpressurized all-terrain vehicle (ATV), and a pressurized rover (PR). It is assumed that there is no resupply or repair and all the spares that may be required during the course of the mission have to be brought in along with other cargo. In the Hubble Space Telescope, in addition to several rate sensors (gyros), a few of its electronic control units (ECU) also failed [4,5]. Thus, as a realistic example an ECU is taken to be the component under consideration here. This ECU is assumed to be reconfigurable such that it can be employed for use in any of the mission elements described above. The QPA of this ECU for the ADV, ATV, HAB, and PR are 1, 1, 3, and 2, respectively. The MTTF of this ECU is assumed to be $100,000 \mathrm{~h}$ of operation (which is a realistic estimate). The failure rate $l$ is then simply the reciprocal of this MTTF. Because this is a Mars mission, a surface stay of 600 days [6] is assumed (therefore the mission time starts at day 1 on the surface and ends at day 600 when the crew departs).

A specific operational scenario is assumed, and the operation time profiles for the elements shown in Fig. 5a. At each time instant, a value of 1 indicates that the element is operating, whereas a value of 

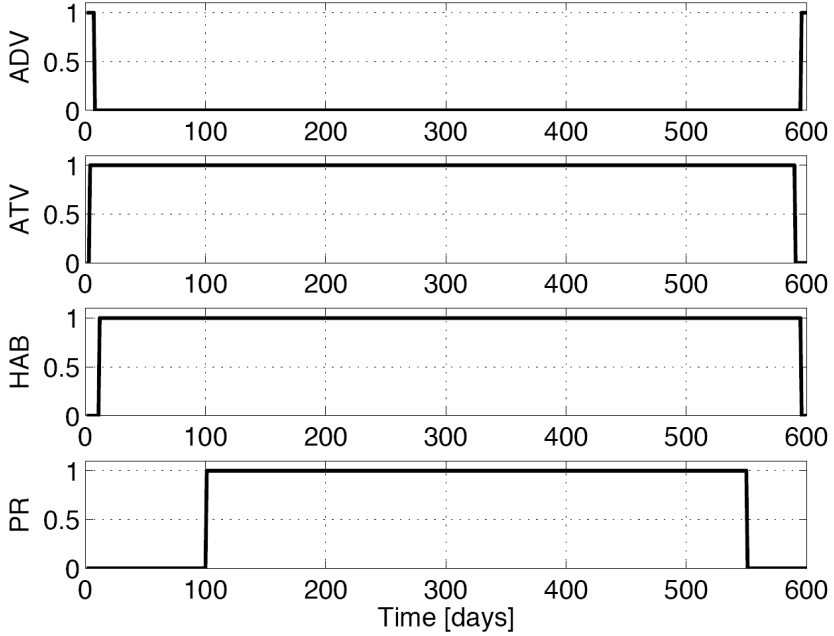

a) Operation time profile

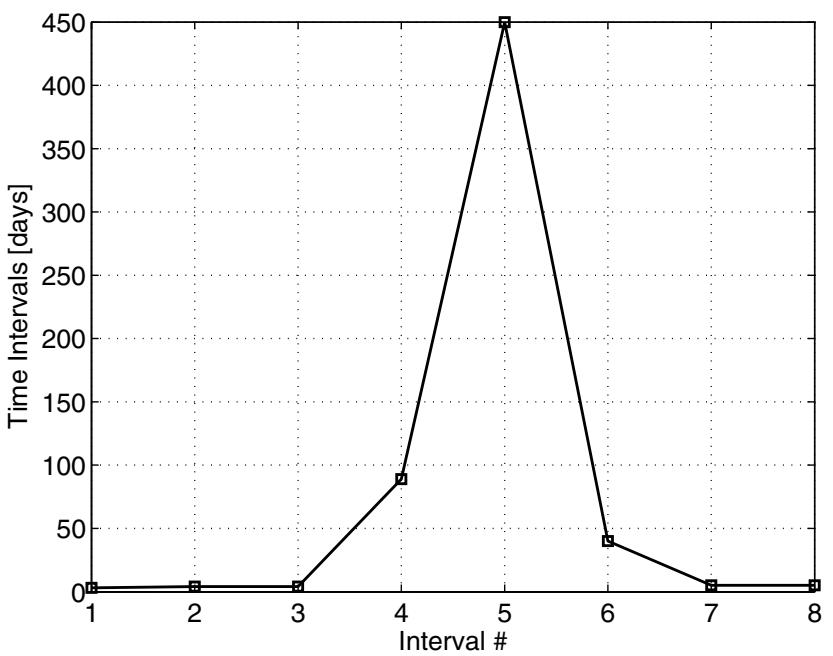

b) Time Intervals $\left(t_{i}-t_{i-1}\right)$

Fig. 5 Operation time profiles of the mission elements.

zero is shown when the element is idle. Based on these operation profiles, the instances of time in which a change in the operating elements occurs are determined.

It can be observed that the time instances in Fig. $5 \mathrm{~b}$ vary widely in duration, with some intervals being very small (a few days), while others are very large (hundreds of days).

As discussed earlier, in the reconfigurable case the system availability may not necessarily be the lowest at the end of the mission because the number of available spares are derived from idle components in addition to a spares repository [Eq. (12)]. This can be seen in this case also where Fig. 6 shows that the availability is lowest in the fifth interval. This interval happens to be the longest and also has the most elements concurrently operating, thus making its $s_{e}$ the lowest (as shown in Fig. 7). Because the potential number of spares are the lowest in this interval, the availability is also the lowest. The availability could have been relatively higher, however, if this occurred earlier in the mission because the chances of part failure would have been lower. As discussed in Eq. (22), this minimum value of availability over the mission duration is considered for comparison with dedicated cases (in which only the end-of-mission availability is evaluated).

In addition to applying the analytical model for this particular case study, a discrete-event simulator was also used to compare the results for the reconfigurable case. Figure 8 shows the outcome of 5000 simulations for an inventory spares level $s_{I}$ of 0 for the reconfigurable case. It can be seen that in some cases, all four elements were not

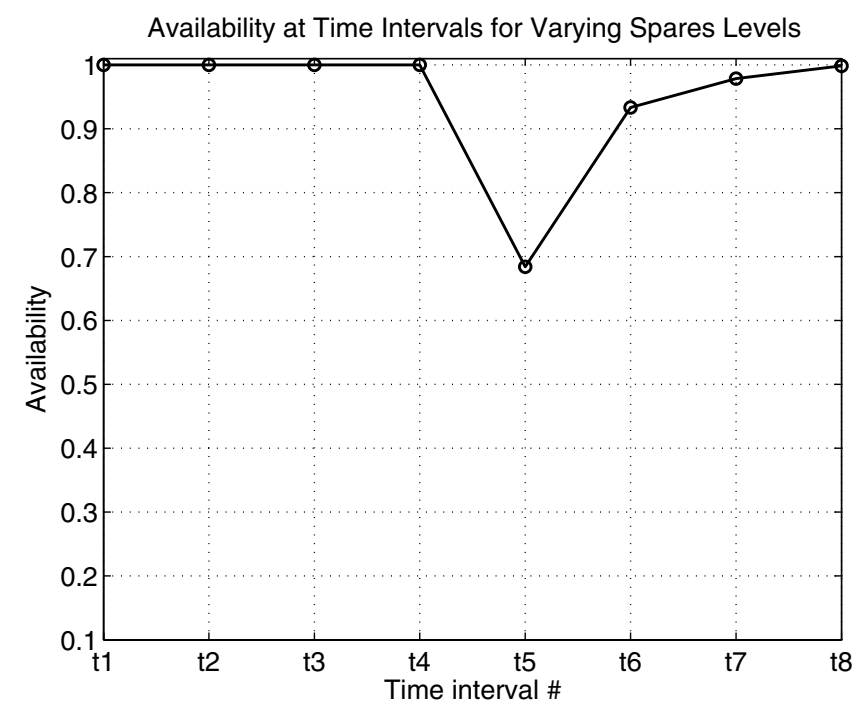

Fig. 6 Availability in various time intervals for the reconfigurable case.

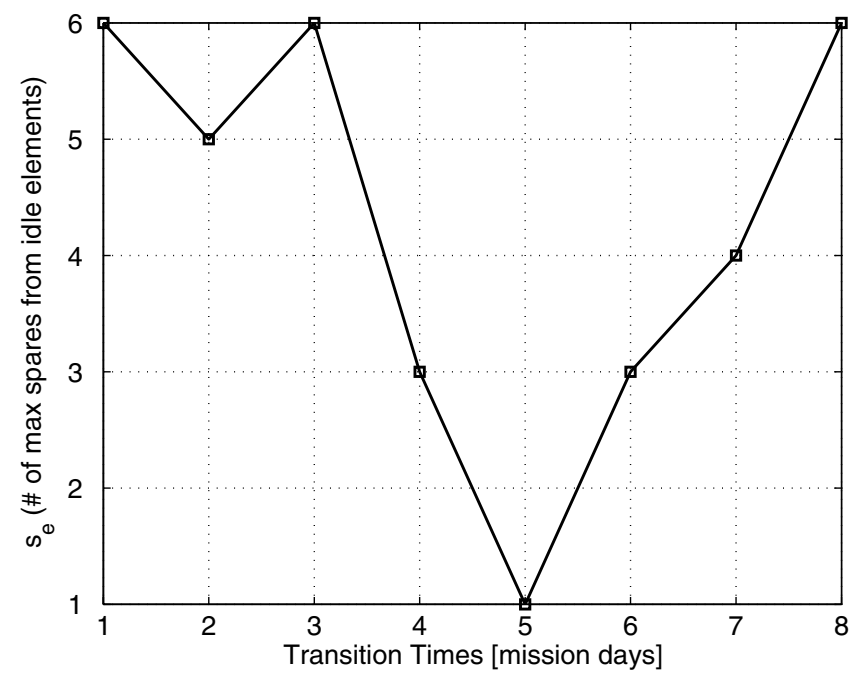

Fig. 7 Maximum spares from idle elements in various time intervals.

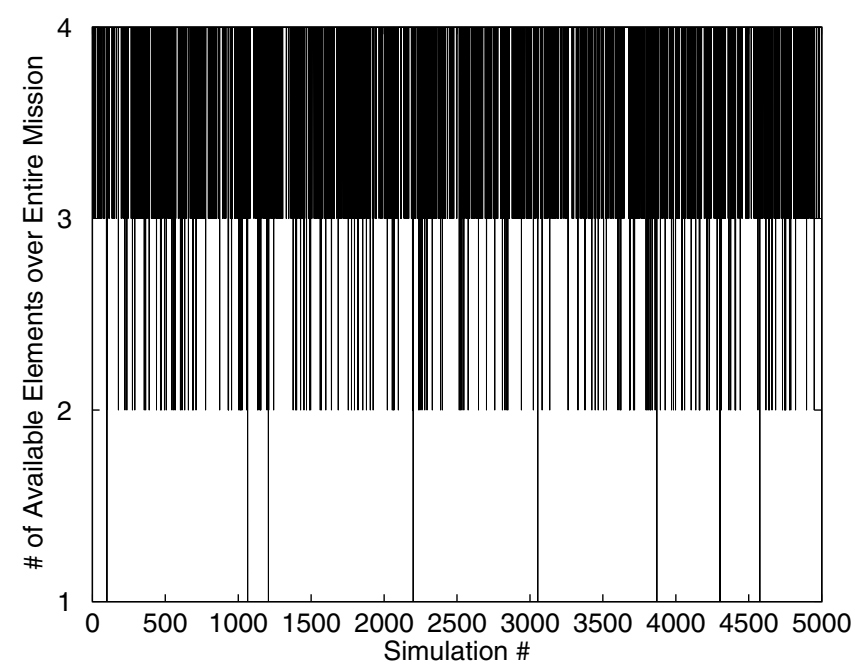

Fig. 8 Simulated results of system availability (5000 runs, $s_{I}=0$ ).

available, despite their ability to use scavenged parts. Overall, the availability was found to be $78.08 \%$ for this particular spares level.

The system availability as a function of inventory spares level $s_{I}$ was then determined using the analytical model [with Eqs. (6) and 
Table 1 Optimal spares allocation for the dedicated case and operational scenario shown in Fig. 5.

\begin{tabular}{lcccc}
\hline \hline Total spares $\left(s_{I}\right)$ & ADV & ATV & HAB & PR \\
\hline 1 & 0 & 0 & 1 & 0 \\
2 & 0 & 0 & 1 & 1 \\
3 & 0 & 1 & 1 & 1 \\
4 & 0 & 1 & 2 & 1 \\
\hline \hline
\end{tabular}

(22)] and the simulator. The system availability for the reconfigurable case was computed in two ways using both the conservative assumption [Eq. (10)], and relatively nonconservative assumption [Eq. (9)], respectively.

In the dedicated case, the assumption was that each element had its own particular ECU which cannot be used in the other elements. Furthermore, for each given spare level, the optimal spares combination (for each element) that maximized the system availability was used. The optimal combination was computed by simply doing a full-factorial analysis (because the number of elements and the spares level were small). All the possible combinations for a total spares level $\left(s_{I}\right)$ to be distributed among the $E$ elements were determined and the corresponding level of availability was computed. The combination that produced the highest overall availability at the mission level was chosen for each particular value of $s_{I}$. So, for instance, if a total of three spares were part of the repository $\left(s_{I}=3\right)$, the optimal combination of having spares for the ADV, ATV, HAB, and PR was determined such that the system availability was highest for the total spare level of 3 . In this case it worked out to be one spare each for the ATV, HAB, and PR, while none for the ADV. Details of the optimal combinations of the dedicated spares are given in Table 1.

Figure 9 shows how the reconfigurable and dedicated cases compare in terms of system availability as a function of initial spares level. A total of four lines, corresponding to a particular case, are shown. The first line from the top is the simulated result obtained for each spares level for the reconfigurable case. The second line from the top shows the analytical result from the model using the nonconservative approach for computing $\lambda_{e}$ [(Eq. (9)]. As expected, it gives a lower prediction for the availability as compared to the simulated case. However, the third line which is the analytical prediction using the conservative value for $\lambda$ [Eq. (10)], is the most stringent in the availability prediction. The fourth line is for the dedicated case and is always lower in availability as compared to the reconfigurable scenario. It should be noted that for the zero spares level case, the reconfigurable scenario can offer significant benefits in terms of increased system availability. It can be expected that spares will not be carried along for all subsystems/components due to overall cargo mass and volume capacity constraints. The advantage

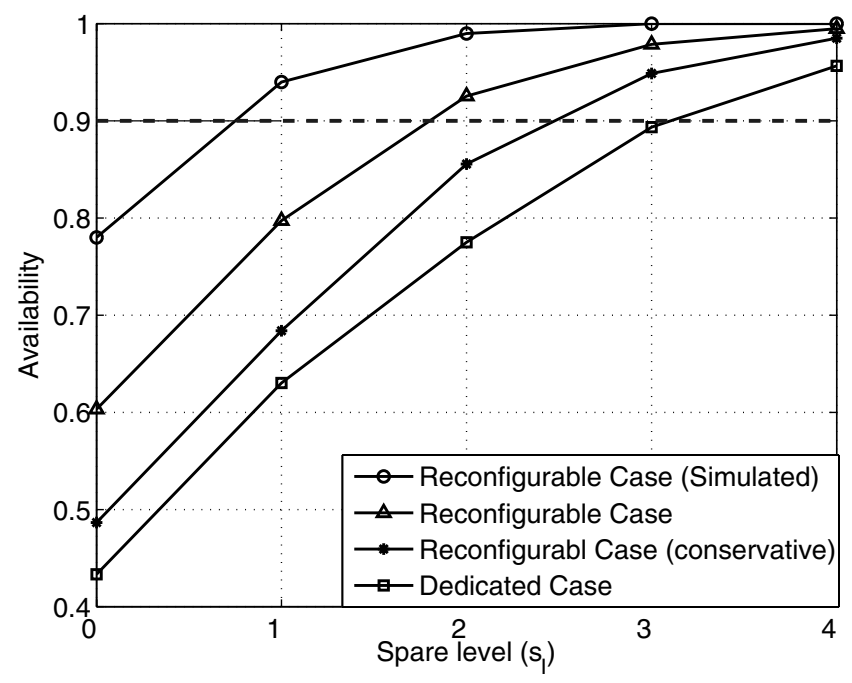

Fig. 9 Availability vs inventory spares levels for reconfigurable/ common and dedicated cases for a simulated 600 day mission. from the reconfigurable case for the zero spares level therefore indicates the benefit that can be achieved for the type of parts for which there will be no spares.

In the case of the Hubble Space Telescope, the mass of its ECUs was $8 \mathrm{~kg}$. If several components in several mission elements are reconfigurable or common, the total mass savings can become significant, or for a given spares mass the system availability can be increased. Plots such as the one shown in Fig. 9 can thus be used to quantify the impact of reconfigurability or commonality on the sparing requirements (and subsequently mass and volume). For instance, in this particular example if a $90 \%$ availability target is to be achieved (dashed line), only one reconfigurable or common spare (as shown for the simulated result), or two spares (as shown for the nonconservative result) are needed versus three spares for the dedicated case. There can thus be a savings of at least $33 \%$ or even $50 \%$ in the number of required spares for a given system availability target.

The advantage in mass and volume can also potentially be traded with component reliability. Extremely stringent reliability requirements are often a significant cost driver in both manned and unmanned missions. Figure 10 shows how the availability compares for different spares levels between the two cases in which the dedicated case has ECUs with MTTF of $100,000 \mathrm{~h}$, while the reconfigurable ECUs have MTTF of 75,000 h. For one spare, the two cases are almost identical, and for more spares the reconfigurable case offers an advantage. The reconfigurable case can allow for lower reliability and therefore potentially lower cost.

The availability of the system as a function of failure rates is also examined using the assumptions of this case study. It was analytically shown in Sec. IV that the availability for the reconfigurable and dedicated case changes at different rates with a changing failure rate $l$. Figure 11 shows how the availability decreases with increasing failure rate (with $s_{I}$ set to 1 ), and graphically shows the tradeoff that exists between the two cases.

Trade studies can therefore be conducted to find the crossover failure rates that make one case favorable over the other. It is interesting to note that reconfigurable/common parts can actually perform worse because of risk pooling when failure rates are large. In that case, a low reliability common part introduces a vulnerability that now affects all elements in the mission, whereas in the dedicated case elements are somewhat protected from such systemic problems by virtue of their separate spare pools. However, if reliability is reasonably good (in this example if $l<0.0006$ ) the reconfigurable case will always be better.

The variation of the availability with $\gamma$ can also be explored by varying the operational scenarios. The scenario shown in Fig. 5 was not used in this computation. Four different scenarios were considered instead, with one, two, three, and all four elements

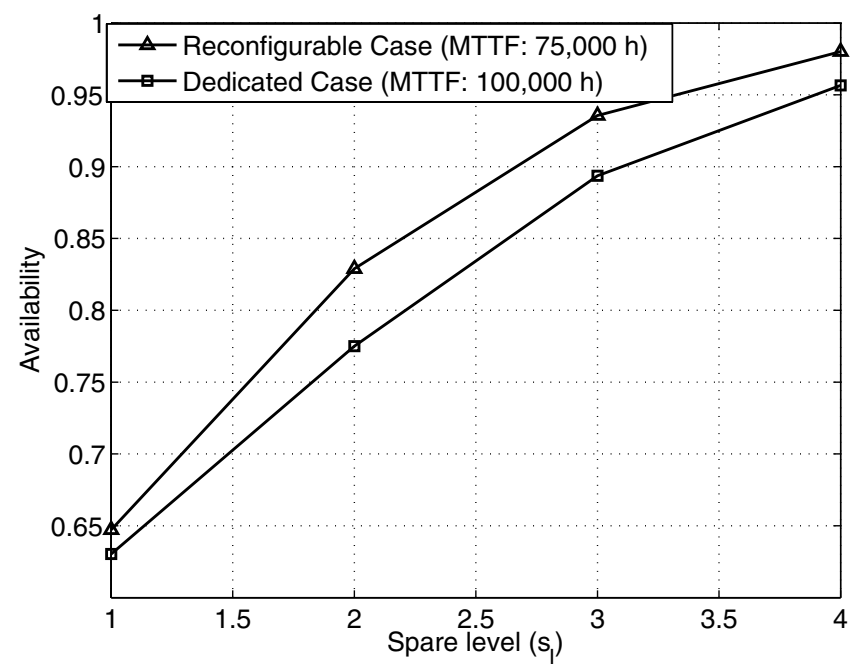

Fig. 10 Availability for reconfigurable versus dedicated spares with different MTTF. 


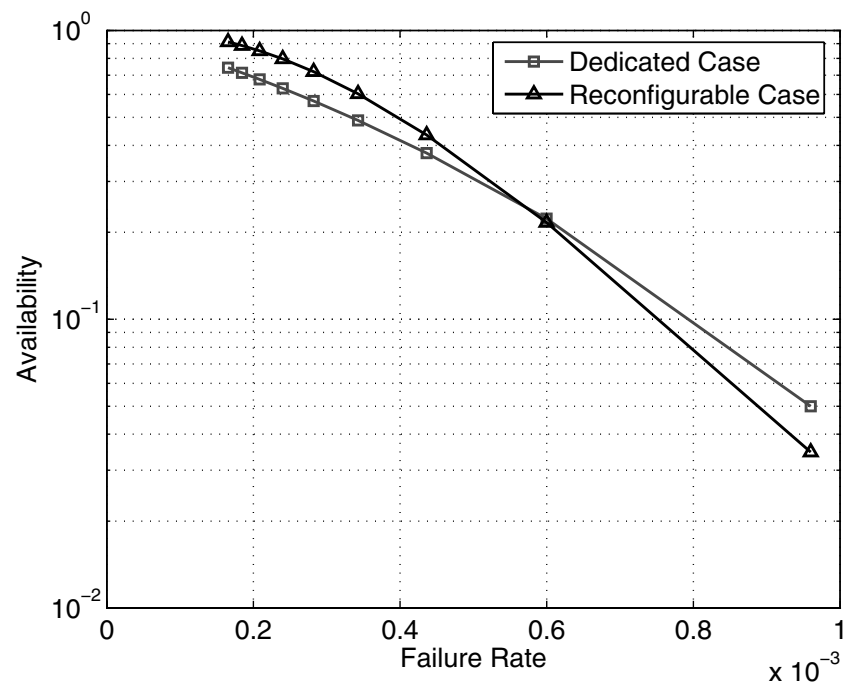

Fig. 11 Availability vs failure rates for reconfigurable and dedicated spares, spares level $s_{I}=1$.

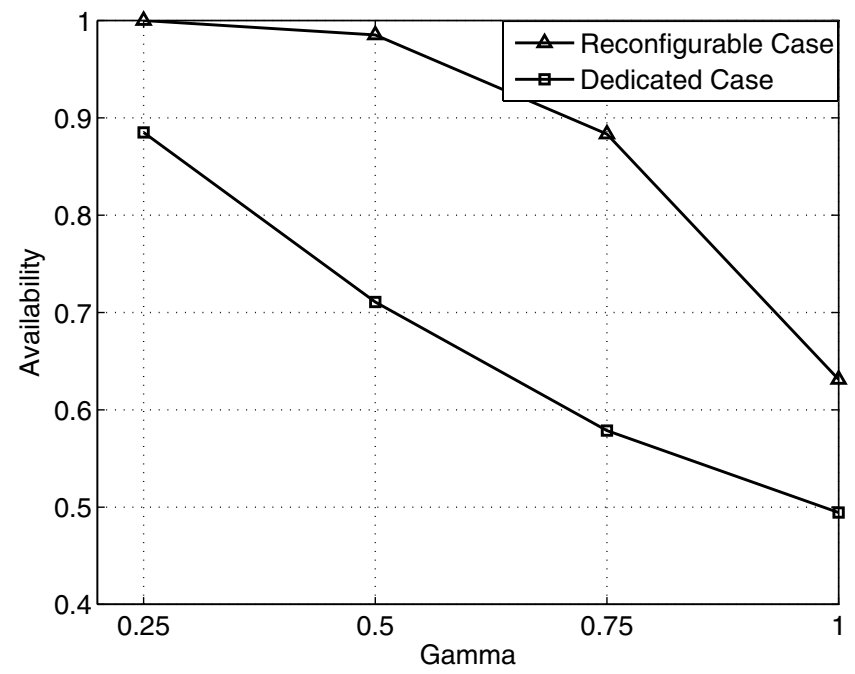

Fig. 12 Availability vs gamma for the reconfigurable case.

operating at a given time, corresponding to a $\gamma$ of $0.25,0.50,0.75$, and 1 , respectively. The inventory spares level $s_{I}$ was set to 2 . Figure 12 shows how the availability decreases with increasing $\gamma$.

It is seen that the availability changes very rapidly for the reconfigurable case with increasing $\gamma$ because there are fewer spares that can be counted in the total spares pool (due to few idle elements). In the four scenarios constructed for the different $\gamma \mathrm{s}$, the total operational time of the individual elements is gradually increased. In the dedicated case, as $\gamma$ increases, there is thus a more gradual decrease in the availability. If the total operational time remains the same between the various $\gamma \mathrm{s}$, then there will be no change in availability [as shown through Eq. (43)] for the dedicated case.

It can also be noted that the reconfigurable case has appreciably higher availability even in the case of $\gamma=1$. This is because although there are no elements that can lend any extra spares (because all elements are operating at all times), the fact that the spares are common means that any failure in any element can be fixed if there is any spare available in the repository. Whereas in the dedicated case, a failure in a particular element can only be fixed if a spare for that particular element is available. Thus, even if there are no elements that can be temporarily cannibalized, reconfigurable (or common) components can offer advantages in terms of required spares for a desired level of system availability.

\section{Conclusions}

This paper develops a model for estimating the effect of having reconfigurability or commonality (among components of different mission elements) on sparing requirements. It is seen from the simple example analyzed in the paper that the capability to cannibalize parts from idle elements when no other spares are available can be very valuable because it allows for the reduction in the total spares requirements. To have this capability, commonality and interchangeability throughout system designs and across mission elements will be needed.

The tradeoff in having such a capability, however, might be that the increased complexity required for each reconfigurable component could result in increased costs and reduced reliability. This model can thus be used in studying tradeoffs between the architectural qualities (of reconfigurability/commonality, etc.) with component reliability and element operational scenarios.

In the present work the equations have been developed for only one type of component, and no in situ repair was allowed. In future studies, extension to the multicomponent case, that is, accounting for the fact that each element is made up of multiple components, will be made. The effect of redundancy within components/elements will also be explored for evaluating the availability. Furthermore, in the reconfigurable case, the effect on crew time due to delays from carrying out the reconfigurations will be examined. The effect of local repair capability (which allows for returning parts to the spares repository) will also be analyzed.

The immediate application of this work is in the context of NASA's Vision for Space Exploration, but we expect that other applications such as logistics for remote outposts on Earth, oildrilling platforms, or flexible manufacturing facilities will also be of interest.

\section{References}

[1] Caglar, D., Li, C. L., and Simchi-Levi, D., "Two-Echelon Spare Parts Inventory System Subject to a Service Constraint," IIE Transactions, Vol. 36, No. 7, 2004, pp. 655-666.

[2] Shishko, R., "Calculating Space Station Resource Prices," AIAA Paper 2000-5322, 2000.

[3] Bachman, T. C., and Kline, R. C., "Model for Estimating Spare Parts Requirements for Future Missions," AIAA Paper 2004-5978, 2004.

[4] Pizzano, F., and Kincade, R., "Hubble Space Telescope Maintenance and Refurbishment Planning Analysis," AIAA and NASA, Symposium on the Maintainability of Aerospace Systems, AIAA, Washingon, D.C., July 1989; also AIAA Paper 1989-5048, 1989.

[5] Fricke, R. W., Jr., "STS-61 Space Shuttle Mission Report," NASA TM110545, 1994, NSTS-08288; also NAS 1.15:110545.

[6] Hoffman, S. J., and Kaplan, D. I., Human Exploration of Mars: The Reference Mission of the NASA Mars Exploration Study Team, NASA Special Publication 6107, 1997. 